\title{
Application of three-dimensional wire localization and orientation in the resection of non-palpable breast lesions
}

\author{
DECHUN LI, JINGJING XU, JIE ZHANG, DANDAN XIA and GUOQING SHAO \\ Department of Radiology, The Affiliated Xuzhou Center Hospital of Nanjing University \\ of Chinese Medicine, Xuzhou, Jiangsu 221009, P.R. China
}

Received January 25, 2016; Accepted August 22, 2016

DOI: $10.3892 / \mathrm{ol} .2017 .6014$

\begin{abstract}
The aim of the present study was to compare the application value of the 3D stereo wire localization technique guided by a full digital breast X-ray machine, and general location technique in the resection of breast non-palpable lesions. A retrospective analysis of the clinical data of 80 patients with abnormal findings for non-palpable breast molybdenum target X-ray was conducted between June, 2011 and July, 2014. The patients were divided into two groups: 40 cases with a general location of lesions identified by perturbation axial + standard lateral radiograph to guide the clinical operation biopsy (group A); and 40 cases with a general location of lesions identified by the 3D stereo wire localization method to guide the clinical operation biopsy (group B). The one-time success rate of the resection of breast lesions of the two groups of patients was compared. Both groups of patients completed the operation. Of the 40 cases in group A, 33 cases had one-time complete resection of lesions, 5 cases had incomplete resection with the second resection, and the lesions of 2 cases were not excised and the one-time success rate of the breast lesion resection was $82.5 \%$. Of the 40 cases in group B, lesions of 38 cases were one-time complete resection and 2 had vagal reaction; thus, the one-time success rate of breast lesion resection being $95 \%$. Compared to group $\mathrm{A}$, the one-time success rate of breast lesion resection of group B was statistically and significantly higher $\left(\chi^{2}=3.638, \mathrm{P}<0.05\right)$. In conclusion, the $3 \mathrm{D}$ stereo wire localization technique guided by the full digital breast X-ray machine for the excision of non-palpable breast lesions has high positioning accuracy with small operation excision scope. It is therefore an effective method that can be
\end{abstract}

Correspondence to: Dr Guoqing Shao, Department of Radiology, The Affiliated Xuzhou Center Hospital of Nanjing University of Chinese Medicine, 199 Jiefang South Road, Xuzhou, Jiangsu 221009, P.R. China

E-mail: ubl651442181nf@163.com

Key words: breast X-ray photography, breast disease, breast plate photography, three dimensional, wire localization utilized for the qualitative diagnosis of non-palpable breast lesions.

\section{Introduction}

Breast cancer has a high incidence of malignant tumors in women and is currently on the increase, with the occurrence age becoming increasingly less $(1,2)$. Color Doppler ultrasound and ultrasound elasticity imaging have an important clinical value for the diagnosis of breast cancer (3). However, clinical diagnosis is difficult for some early stage breast cancers without abnormality in the non-palpable mass ultrasound examination and with only manifestations of cluster calcification, structure distortion, asymmetric density or small nodular changes in mammography (4).

Previous findings showed that 3D X-ray stereo wire localization biopsy operation has high precision, with a diagnostic accordance rate of $90.9 \%$, and an accurate early breast cancer diagnosis rate of up to $100 \%$ (5), which is useful for clinicians in the selection of an effective treatment method.

In the present study, a retrospective analysis of the clinical data of 80 patients with non-palpable breast lesions diagnosed using molybdenum target X-ray at the Xuzhou Hospital Affiliated to Southeast University (Jiangsu, China) between June, 2011 and July, 2014, was performed with a comparative analysis of one-time success rate of lesion resection by general positioning operation biopsy and 3D stereo wire localization biopsy.

\section{Materials and methods}

Subjects. A total of 80 cases diagnosed according to the preoperative positioning methods were divided into 40 cases of rough positioning (group A) and 40 cases of 3D solid wire localization (group B). The inclusion criteria for the study were: clinical palpation without finding lesions of small sand-like calcification, suspicious clustered calcifications, structure distortion, asymmetric dense and suspicious nodules only in molybdenum target X-ray photography; on the basis of the standard classification (6) of breast imaging reporting and data system, BI-RADS of USA Radiological Society, the patients of class BI-RADS-4 and class BI-RADS-3 (required lesion biopsy for their anxiety). The exclusion criteria for the study were: indeterminate class BI-RADS-0 that needed 
further imaging examination; class BI-RADS-1, 2 normal and benign lesions; part of class BI-RADS-3 patients who agreed on 6-month follow-up review; and class BI-RADS-5 diagnosed with breast cancer.

The molybdenum target X-ray film was read by two experienced physicians for the diagnosis of breast imaging on the special workstation. Lesions were classified on the basis of the BI-RADS standard classification, and if there were differences of opinion, case discussion was expanded in the department until the classification results were consistent.

Patients characteristics. Patients in group A were 25-61 years (average age, $44 \pm 5$ years). In the molybdenum target X-ray examination group, there were 24 cases of sand-like calcification, 8 cases of small suspicious distortion, 5 cases of clustered calcifications, and 3 cases of asymmetric structure of dense and suspicious nodules. The patients in group B were 28-62 years (average age, $45 \pm 4$ years). The molybdenum target X-ray examination revealed 26 cases of sand-like calcification, 7 cases of small suspicious distortion, 5 cases of clustered calcifications, and 2 cases of asymmetric structure of dense and suspicious nodules. There was no statistically significant difference in the age $(\mathrm{t}=-0.348, \mathrm{P}=0.367>0.05)$ and results of molybdenum target $\mathrm{X}$-ray examination $(\mathrm{U}=7.5, \mathrm{P}=0.885>0.05)$ between the patients of the two groups.

Instruments and equipment. Full digital breast X-ray machine and the supporting 3D stereotactic system (Hologic, Bedford, MA, USA), and double hook wire localization needle (Budd Co., Philadelphia, PA, USA) were used for imaging.

Methods. The patients underwent routine mammography axial, lateral and standard mediolateral oblique radiography.

General positioning method. Each breast was regarded as a clock dial, and according to the preoperative routine mammography axial and standard lateral image, the location of the lesion in the quadrant and the time range of the corresponding clock disc was assessed. Adobe Photoshop CS version 10.0 (Adobe Systems, Inc., San Jose, CA, USA) was used to measure the imaging distance from the nipple to the papillary lesions, and the reports were filled operating physicians for surgical biopsy.

$3 D$ stereo wire localization method. Patients were required to be seated. A puncture rack was installed, and the lesion puncture was placed into the placement box, radiography was assessed and the lesions in the operational range were adjusted (Fig. 1), and fixed by oppression. The position of the needle tip was calibrated, and the coordinates were 0 .axis $Z$. Perturbation of $+15^{\circ}$ and $-15^{\circ}$ film was determined in the digital spot mammography, the target lesions display system was selected, and the puncture point $\mathrm{x}, \mathrm{y}$ and $\mathrm{z}$ axes were accurately calculated by computer to determine the needle point and needle depth. Regular disinfection, and local anesthesia, were used and the needle puncture was inserted, and radiography confirmed the correct position (Fig. 2). The needle sheath, and indwelling double hook wire was pulled out (Fig. 3), and the body part of the guide wire was excised and fixed by ligule tape winding.

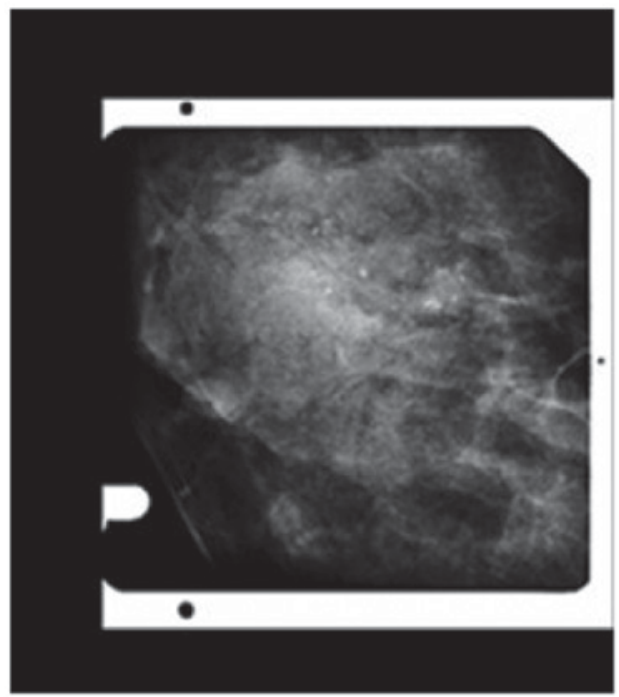

Figure 1. Breast calcification in the positioning frame

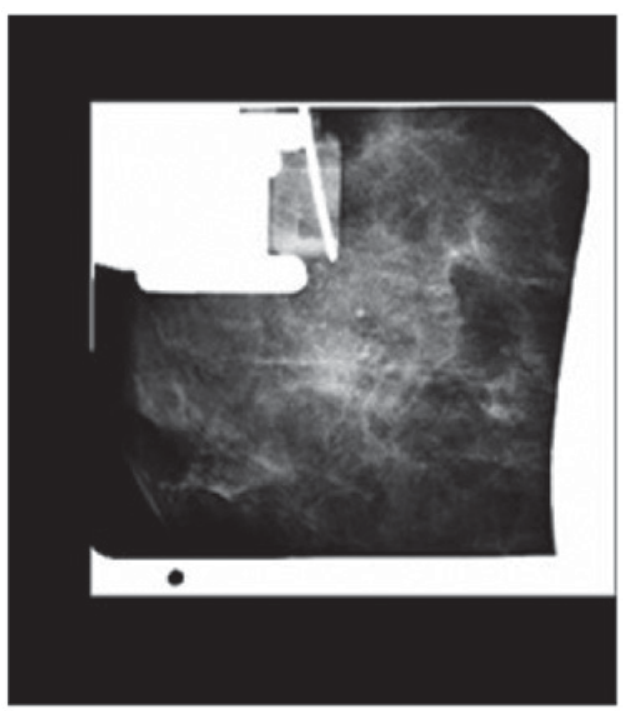

Figure 2. The positioning needle was inserted, and the needle tip is located in the focal region.

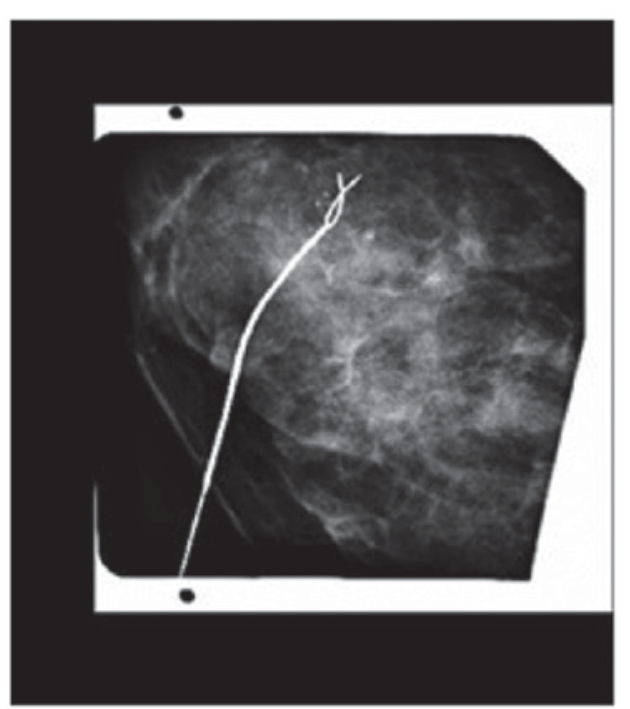

Figure 3. Indwelling double hook wires in the lesion area. 


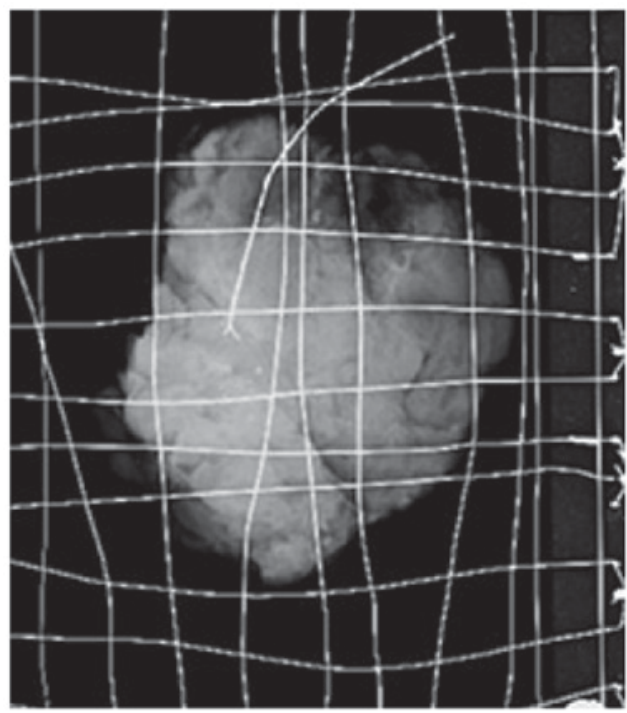

Figure 4. Postoperative specimen shows a one-time completed resection of lesions.

Biopsy operation. At the general location of the lesion, the surgeons made incisions according to the focus range provided by the radiologists. The operation excision range was generally based on the lesion size. For the lesions localized by 3D stereo wire, the surgeons followed guided wire localization to excise the lesion biopsy; with surgical resection being generally in the range of $20 \mathrm{~mm}$ around the tip of localization wires. Two groups of cases were marked long, short, and single, or double line of resection specimens based on the direction and position in the operation process, and then the specimen was sent to the radiology radiograph. The radiologist compared specimen radiography and preoperative axial and lateral radiographs, and observed the resected specimen to determine whether lesions were completely contained. If the lesion size, quantity, and scope were consistent with previous X-ray film, it was considered as completely removed (Fig. 4), and the needle head was inserted into the pathological lesions. However, if the lesion was not completely removed according to the position of marked lines, the physician resected the remaining lesions, and the specimens were sent to the Department of Pathology.

Statistical analysis. Data were analyzed using statistical software SPSS 16.0 (Chicago, IL, USA). The measurement data of approximate normal distribution are presented as mean $\pm \mathrm{SD}$, and the groups were compared by the independent sample Student's t-test. Measurement data were obtained using the Mann-Whitney $U$ test, $\chi^{2}$ test and non-parametric test. $\mathrm{P}<0.05$ was considered as the difference with statistical significance.

\section{Results}

Of the 40 cases of group A, 33 cases completed one-time resection of lesions (82.5\% success rate), of which, 5 cases were partially removed, 3 cases underwent a second total resection, and 2 cases did not cut to the lesion, even after six months of repositioning of resection. Of the 40 cases of group B, 38 cases completed one-time resection of lesions (95\% success rate),
2 patients had vagal reaction, and were required to rest for a few minutes, prior to completing positioning with successful resection.

As the moving range was extremely large prior to the operation, the biopsy resulted in the guide wire moving, and the radiologist resected the incomplete resection a second time in two patients. These results showed that the success rate of one-time complete lesion resection of group B was significantly higher than that of group A (95 vs. 82.5\%), and the difference was statistically significant $\left(\chi^{2}=3.638, \mathrm{P}<0.05\right)$.

\section{Discussion}

The performance of specific imaging of non-palpable breast lesions is poor with difficulties for the differentiation of benign, and malignant lesions (7). Prior to the introduction of the $3 \mathrm{D}$ stereotactic localization system for the non-palpable lesions that were only displayed using mammography, the traditional general clinical positioning methods were used to provide the location of the lesion to surgeons. General location requirements for the technical level of radiologists and surgeons are higher, and the positioning range as well as the operation excision range are larger. At the same time, because the position in the operation is different from the one in the film with the breast oppression image, accurate resection of the lesion becomes difficult due to a deviation in the location of the lesion during surgery.

At present, some hospitals use molybdenum target $2 \mathrm{D}$ guide wire localization technique, known as $2 \mathrm{D}$ wire localization. It can just provide $\mathrm{x}$ and $\mathrm{y}$ axes of the target lesions, but the determination of $\mathrm{z}$ axis, the value of the depth of the needle requires the experience of radiologists, and is bound to cause a distance error between the tip and target lesion to expand the scope of operation (8). Although the $2 \mathrm{D}$ wire localization technique is relatively more accurate than that of general positioning, the depth of the needle is easily influenced by subjective factors, and is less accurate than 3D stereo positioning. Evidence has shown that, the accuracy of 2D guided wire localization and the comfort it brings to the patients is lower than that of 3D stereo wire localization (8).

The 3D stereotactic localization system has been developed based on 2D wire localization, but adds another dimension. Additionally, through the computer the exact 3D coordinates of target lesions can be calculated for more accurate positioning. It can also determine the resection range of lesions as well as smaller resection range, once the guide wire is placed by the surgeon and there is less damage to the normal breast tissue. The 3D stereo wire localization technique guided by the molybdenum target may identify an earlier palpation-negative lesion that can only be found in the $\mathrm{X}$-ray examination through guide wire orientation to direct clinicians during excision and the pathological examination. This is important in the precise excision of the lesion, which can further improve the earlier diagnostic rate of breast cancer and the accurate rate of resection (9).

To determine the shortest distance from the lesion to the surface is particularly important to minimize the damage to the mammary gland during surgery. Finding the shortest path can reduce normal glandular tissue of the guide wire and resected tissue during surgery. To achieve this, we improved 
the manner in which the axial needle was inserted in different positions selected for different puncture directions, taking the comfort of patients into consideration. An axial needle was used for the lesions generally located in the inner and outer quadrant; for the lesion located in the lateral or medial quadrant, with the puncture rack being rotated to the position of $90^{\circ}$; whereas for lesions under the internal or external quadrant, the puncture rack was generally rotated to $45^{\circ}$ or $60^{\circ}$.

As the precision requirements of $3 \mathrm{D}$ stereo wire localization technique are higher, authors suggest that in the course of the operation the following points remain to be clarified: i) after fixing the lesions, patients should remain stationary during operation; ii) because the patients are seated and see the surgical procedure, a few patients may have vagal reaction, and the clinical manifestations thereof are dizziness, pale, rapid heartbeat, sweating and weakness in the limbs (9). At that time, patients should be allowed to relax or rest in supine position for a few minutes. Most of the patients recovered by themselves to complete the positioning operation; severe clinical manifestations were alleviated after injection of atropine and patients continued until completion of the positioning operation, or returned on another day. To avoid the vagal reaction of patients before and during surgery because of nervousness, communication should be constant with the patient in order to relieve the tension; iii) since breast compression is needed for the guide wire localization, it is not appropriate to use this for patients with extremely small and thin breasts; iv) it is not appropriate to use wire localization for the patients whose lesion location is extremely high and close to the chest wall, and by adjusting the gantry angle whose lesion cannot be placed in the positioning frame; and v) although the guide wire localization technique operation is simple and safe, there is a possibility of hematoma around the location points. Guide wire localization technique should also not be used for patients with coagulation dysfunction.

In conclusion, the 3D stereo wire localization technique guided by the full digital breast X-ray machine has an important diagnostic value for the palpation-negative breast lesions, as it can be useful for earlier identification, diagnosis and treatment of breast lesions, and through various technical improvements, positioning may provide a more accurate image with smaller operation excision scope (10). This meets the demand for esthetic beauty and quality of life of women and is worthy of clinical application and promotion. As the study time was short and a small sample size was included in the present study, there are certain limitations to the use of the wire localization technique for the cases whose images had structural distortion and were asymmetrically dense. Future studies may identify more useful ways of conducting biopsy for the abovementioned cases.

\section{References}

1. Ly M, Antoine M, Dembélé AK, Levy P, Rodenas A, Touré BA, Badiaga Y, Dembélé BK, Bagayogo DC, Diallo YL, et al: High incidence of triple-negative tumors in sub-saharan Africa: a prospective study of breast cancer characteristics and risk factors in Malian women seen in a Bamako university hospital. Oncology 83: 257-263, 2012.

2. Mao Z, Zhu Z, Wang H, Han W and Zhao Y: Modified radical mastectomy with Auchincloss method for 65 cases of breast cancer. Chin J Anat Clin 19: 313-315, 2014 (In Chinese).

3. Yin R, Yan S and Ling X: Diagnostic value of color Doppler ultrasound and $\mathrm{X}$-ray mammography in the early breast cancer. Chin J Anat Clin 19: 299-302, 2014 (In Chinese).

4. Zhang H, Liang F, Jia Z, Jiao J, Peng Y and Yu Z: Diagnosis and therapy of palpation negative breast lesion. China J Mod Med 18: 3517-3519, 2008 (In Chinese).

5. Chadwick DR and Shorthouse AJ: Wire-directed localization biopsy of the breast: an audit of results and analysis of factors influencing therapeutic value in the treatment of breast cancer. Eur J Surg Oncol 23: 128-133, 1997.

6. Cardeñosa G: Breast Imaging Companion. 3rd edition. Lippincott Williams \& Wilkins, Philadelphia, PA, pp465-467, 2008.

7. Feng RD, Lu J, Li R, Deng Z and Gao K: Preoperative wire guided localization under X-ray guidance for nonpalpable breast lesions. Chin J Med Imaging 21: 341-343, 2013 (In Chinese).

8. Zhao H, Yin C and Zhao A: Comparison of two-dimensional and three-dimensional $X$-ray guided wire localization breast biopsy. Zhongguo Jieru Yingxiang Yu Zhiliaoxue 8: 307-309, 2011 (In Chinese)

9. Fu Y, Ji Z, Ding W, Ye F and Lou C: Thermoacoustic imaging over large field of view for three-dimensional breast tumor localization: a phantom study. Med Phys 41: 110701, 2014.

10. Yang G, Zhang J, Hao X, Chang Y, Zhang S, Yang S and Wei P: Application of 3D X-ray guided wire localization of non-palpable lesion with microcalcification in breast. J Pract Radiol 29: 752-754, 2013 (In Chinese). 